\title{
Cultivo de Penicillium spp. em resíduos da colheita de soja para produção de celulase, protease e amilase ${ }^{1}$
}

\author{
Joice Raísa Barbosa Cunha², Fernanda Castro Pires dos Santos ${ }^{2}$, \\ Fábia Giovana do Val de Assis ${ }^{3}$, Patrícia Lopes Leal ${ }^{2}$
}

$10.1590 / 0034-737 X 201663050002$

\section{RESUMO}

O objetivo deste estudo foi avaliar a produção de enzimas amilolíticas, celulolíticas e proteolíticas pela linhagem Penicillium spp. LEMI A8221 cultivada em estado sólido em resíduos da colheita de soja, ao longo de quatro dias, em diferentes condições de $\mathrm{pH}(5,0$ e 6,0$)$, temperatura $\left(30\right.$ e $\left.35^{\circ} \mathrm{C}\right)$ e concentrações de substrato $(70$ e $90 \% \mathrm{p} / \mathrm{v})$. As atividades máximas obtidas para $\alpha$-amilase, $\beta$-amilase, CMCase e protease foram de 0,$20 ; 0,13 ; 0,65$ e $147 \mathrm{U} \cdot \mathrm{mg}^{-1}$, respectivamente. As condições de fermentação influenciaram a atividade das enzimas, sendo a concentração de substrato, a variável mais significativa para o processo. O tempo de fermentação exerceu efeito apenas para as atividades de $\beta$-amilase e CMCase, sendo registrados os menores valores de atividade para essas enzimas, nas primeiras 24 e 48 horas de fermentação, respectivamente. Conclui-se que o isolado Penicillium spp. LEMI A8221 pode ser considerado promissor agente biológico, com aplicação industrial, e o resíduo de soja apresentou-se como fonte de carbono alternativa, no cultivo em estado sólido, para produção de enzimas por esta linhagem microbiana.

Palavras-chave: resíduo agroindustrial; enzimas; fungos filamentosos.

\section{ABSTRACT}

\section{Cultivation of Penicillium spp. in soy bean crop residues for production of cellulase, protease and amylase}

The aim of this study was to quantify the enzymatic activity of amylolytic, cellulolytic and proteolytic enzymes produced by the fungi strain Penicillium spp. LEMI A 8221 under solid state culture in soybean crop residues, during 4 days, in different conditions of $\mathrm{pH}\left(5,0\right.$ and 6.0), temperature $\left(30\right.$ and $\left.35^{\circ} \mathrm{C}\right)$ and substrate concentration $(70$ and $90 \%$ $\mathrm{p} / \mathrm{v}$ ). The maximum activity obtained for $\alpha$-amylase, $\beta$-amylase, protease and CMCase were $0.20 ; 0.13 ; 0.65$ and 147 ${\mathrm{U} . \mathrm{mg}^{-1}}^{-1}$, respectively. The fermentation conditions influenced the enzyme activity, being substrate concentration, the most significant variable to the process. The fermentation time exerted effect only for the activities of CMCase and $\beta$ amylase being recorded smaller values for these enzymes in the first 24 to 48 hours of fermentation, respectively. It is concluded that the isolated Penicillium spp. LEMI A8221 can be considered a promising biological agent with industrial application and soybean residue was presented as an alternative carbon source in solid-state cultivation for enzyme production by microbial strain.

Key words: agroindustrial residue; enzymes; filamentous fungi.

\footnotetext{
Submetido em 28/04/2015 e aprovado em 06/04/2016.

${ }^{1}$ Este trabalho é parte do trabalho de conclusão de curso da primeira autora.

${ }^{2}$ Universidade Federal da Bahia, Vitória da Conquista, Bahia, Brasil. joice.raisa@gmail.com; fe.castro_@hotmail.com; lealpat@yahoo.com.br

${ }^{3}$ Universidade Federal de Viçosa, Departamento de Microbiologia, Viçosa, Minas Gerais, Brasil. fabi_zoo@yahoo.com.br

*Autora para correspondência: lealpat@yahoo.com.br
} 


\section{INTRODUÇÃO}

A preocupação com a utilização de resíduos da agroindústria é crescente, pois estes representam uma das mais importantes fontes renováveis de energia disponíveis no planeta e, quando indevidamente descartados ou utilizados, tornam-se fontes adicionais de poluição ambiental (Saidelles et al., 2012).

Neste sentido, tem-se intensificado o aproveitamento desses materiais como substratos alternativos no desenvolvimento de vários bioprocessos para a produção de diversas moléculas com alto valor agregado, como proteínas microbianas, ácidos orgânicos, etanol, enzimas e metabólitos secundários biologicamente ativos (Sánchez, 2009).

Dentre os resíduos da agroindústria produzidos no Brasil, destacam-se os da colheita da soja (grãos pequenos e quebrados, vagens, hastes e folhas) que é proporcional à produção nacional do grão (Carvalho, 1992). Graças à composição química geral dos resíduos da colheita da soja ( $50 \%$ de celulose, $25 \%$ de hemicelulose e $25 \%$ de lignina), eles podem se apresentar como excelente substrato para o crescimento e desenvolvimento de microorganismos, a fim de se promover a bioconversão da celulose e da lignina em produtos de interesse econômico (Silva et al., 2005).

Especificamente, para produção de enzimas de valor agregado, a técnica de cultivo em estado sólido vem tomando espaço entre as pesquisas, principalmente para produção de enzimas envolvidas na degradação de polímeros vegetais complexos, comumente presentes em resíduos agroindustriais (Couto \& Sanromán, 2005). Wisniewski et al. (2010) verificaram efeito positivo do farelo de soja como substrato suplementar aos resíduos do processamento de cerveja, para a produção de $\alpha$-amilase (0,48 - 12,71 U.g-1), por Macrocybe titans, sob fermentação em estado sólido (FES); Brijwani \& Vadlani (2011) utilizaram casca de soja, sem e com tratamento prévio, como substrato em FES, para produção de enzimas celulolíticas, por Trichoderma reesei e Aspergillus oryzae, e destacaram, dentre os resultados, a produção de endocelulose (47,10 U.g ${ }^{-1}$ de casca de soja tratada) por de T. reesei; Paris et al. (2012) compararam a produção de diferentes tipos de complexos enzimáticos, por FES, utilizando diferentes tipos de sojas (orgânica, transgênica e convencional) com o fungo Aspergillus niger e verifica-

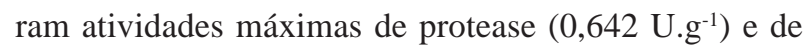

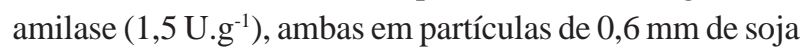
convencional, em 144 h; Garcia et al. (2015) empregaram farelo de soja como substrato, sob FES, e verificaram a

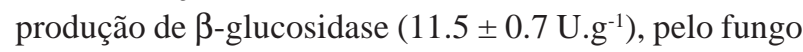
Lichtheimia ramosa; Thakur et al. (2015) também utilizaram farelo de soja para o cultivo de Aspergillus Oryzae, sob FES, e registraram atividade proteolítica, variando entre 1.254 a $1.387,6{\mathrm{U} . \mathrm{g}^{-1}}^{-1}$, sob condições otimizadas de fermentação.

Objetivando-se a produção de enzimas sob cultivo em estado sólido, algumas considerações se fazem importantes, como a seleção do micro-organismo e a determinação das condições ambientais ideais para o processo. Os fungos são os organismos usualmente empregados nesse tipo de cultivo para produção de enzimas, sendo os gêneros Trichoderma, Penicillium, Aspergillus e Humicola amplamente estudados (Sukumaran et al., 2005; Kim et $a l ., 2014)$. Os principais fatores ambientais que afetam a síntese de enzimas microbianas num sistema de cultivo em estado sólido incluem a seleção e a concentração do substrato, o controle da umidade, a temperatura de incubação e o pH, o período de cultivo, a aeração, a agitação e os nutrientes adicionais, que influenciam, significativamente, o crescimento celular e a formação de produtos (Sohail et al., 2009; Saxena \& Singh, 2011).

Sendo assim, este trabalho teve como objetivo avaliar a produção de enzimas amilolíticas, proteolíticas e celulolíticas pela linhagem Penicillium spp. LEMI A8221, sob cultivo em estado sólido, utilizando resíduos da colheita da soja como substrato, ao longo de quatro dias de incubação, em diferentes tempos de cultivo, concentrações do substrato, valores de $\mathrm{pH}$ e temperatura.

\section{MATERIAL E MÉTODOS}

A linhagem fúngica utilizada foi a Penicillium spp. LEMI A8221, oriunda da coleção de culturas microbianas do Laboratório de Enzimologia e Microbiologia Industrial, da Universidade Federal da Bahia, campus Anísio Teixeira, em Vitória da Conquista, BA. A linhagem fúngica foi isolada da rizosfera da cactácea Melocactus sp. e sua reativação foi realizada mediante o cultivo em placas de Petri, com meio de cultura batata-dextrose-ágar (PDA HIMEDIA pH 5,02), por 48 horas.

O experimento foi conduzido conforme delineamento inteiramente casualizado, disposto em arranjo fatorial com dois níveis dos fatores $\mathrm{pH}(5,0$ e 6,0), temperatura (30 e 35 ${ }^{\circ} \mathrm{C}$ ) e concentração de substrato/umidade (70 e $90 \%$ p/v) para a máxima produção de enzimas pelo isolado fúngico sob cultivo em estado sólido em resíduos da colheita da soja (Tabela 1).

O resíduo da colheita de soja foi composto por grãos, pequenos e quebrados, vagens, hastes, folhas e cascas. Cada componente foi submetido à secagem em estufa, a $65{ }^{\circ} \mathrm{C}$, por $72 \mathrm{~h}$, e moído em moinho de facas, sem granulometria definida. Volumes proporcionais de cada componente compuseram o substrato orgânico, que foi autoclavado e utilizado para o cultivo, em estado sólido, da linhagem Penicillium spp. LEMI A8221. 
O cultivo microbiano foi estabelecido em frascos erlenmeyers de $125 \mathrm{~mL}$ com $50 \mathrm{~mL}$ de meio mínimo (1 g.L${ }^{1}$ de sulfato de amônio; 2 g. $\mathrm{L}^{-1}$ de fosfato de potássio; 2,8 g. $\mathrm{L}^{-1}$ de fosfato de sódio; 0,05 g.L $\mathrm{L}^{-1}$ de citrato de ferro; $0,002 \mathrm{~g} . \mathrm{L}^{-1}$ de sulfato de magnésio; $0,01 \mathrm{~g} . \mathrm{L}^{-1}$ de extrato de levedura) e resíduos da colheita de soja, de forma a se obterem as concentrações previamente estabelecidas, de 70 e $90 \%$ p/v, em relação ao volume final. $\mathrm{O}$ pH do substrato enriquecido foi ajustado entre 5 e 6 , utilizando $\mathrm{HCl}$. A inoculação com o isolado fúngico foi realizada com a adição de pequenos discos de meio de cultura, com micélio fúngico, segundo metodologia de Sena et al. (2006). Após inoculação, os erlenmeyers foram incubados em estufas de 30 e $35^{\circ} \mathrm{C}$, ao longo de quatro dias. O experimento foi conduzido com duas repetições para cada tratamento.

A extração do complexo enzimático de cada tratamento ocorreu em intervalos de 24 h de cultivo. Após máxima homogeneização dos conteúdos dos frascos, $3 \mathrm{~g}$ de amostra de cada tratamento foram coletadas e $15 \mathrm{~mL}$ de água destilada foram adicionados, sem agitação, durante 30 minutos. Após filtração, seguida de centrifugação a 10.000 g para a remoção dos sólidos suspensos, coletou-se $1 \mathrm{~mL}$ do extrato resultante, que foi transferido para microtubos, com $10 \mu \mathrm{L}$ de azida sódica (1\%), e acondicionadas em câmara fria $\left(-20^{\circ} \mathrm{C}\right)$, até o momento da quantificação da atividade das enzimas.

Para a quantificação da atividade de $\alpha$-amilase, utilizaram-se $40 \mu \mathrm{L}$ de tampão acetato de sódio (500 mmol.L ${ }^{-1}$, pH 6,0), $100 \mu \mathrm{L}$ de solução de amido $0,5 \%$ (m/v) e $60 \mu \mathrm{L}$ da alíquota retirada do meio fermentativo. Os tubos com essa mistura foram incubados a $40^{\circ} \mathrm{C}$, por 30 minutos, e retirados para adição de $200 \mu \mathrm{L}$ de solução iodo/iodeto 1:1 (v:v), previamente preparada, e $200 \mu \mathrm{L}$ de solução de ácido acético, para se efetuar a paralização da reação. Adicionou-se à mistura água destilada, para atingir o volume final de $10 \mathrm{~mL}$, e procedeu-se à leitura em espectrofotômetro com comprimento de onda de $540 \mathrm{~nm}$ (Miller, 1959).

Tabela 1: Modelo do delineamento experimental fatorial completo, utilizado para as análises dos efeitos dos fatores $\mathrm{pH}$, temperatura e concentração de substrato na atividade das enzimas hemicelulolítica, proteolítica e amilolítica, obtidas a partir do cultivo, em estado sólido, em resíduos da colheita da soja

\begin{tabular}{lccc}
\hline Tratamentos & $\mathbf{p H}$ & $\begin{array}{c}\text { Temperatura } \\
\left({ }^{\circ} \mathbf{C}\right)\end{array}$ & $\begin{array}{c}\text { Concentração de } \\
\text { substrato }(\% \mathbf{~ p / v})\end{array}$ \\
\hline T1 & 5 & 30 & 70 \\
T2 & 5 & 30 & 90 \\
T3 & 5 & 35 & 70 \\
T4 & 5 & 35 & 90 \\
T5 & 6 & 30 & 70 \\
T6 & 6 & 30 & 90 \\
T7 & 6 & 35 & 70 \\
T8 & 6 & 35 & 90 \\
\hline
\end{tabular}

Para quantificação de $\beta$-amilase, utilizaram-se $40 \mu \mathrm{L}$ de tampão acetato de sódio (500 mmol. L $\left.\mathrm{L}^{-1}, \mathrm{pH} 6,0\right), 100 \mu \mathrm{L}$ de solução de amido $0,5 \%$ (m/v) e $60 \mu \mathrm{L}$ da alíquota retirada do meio fermentativo. Os tubos com essa mistura foram incubados a $40^{\circ} \mathrm{C}$, por 30 minutos, e retirados para adição de $800 \mu \mathrm{L}$ de solução de ácido dinitrosalicílico (DNS) a $2 \%$, previamente preparada. A mistura foi fervida por cinco minutos e, ao fim, adicionaram-se 9,0 mL de água destilada, seguindo-se a homogeneização. A atividade enzimática foi obtida a partir da leitura em espectrofotômetro a um comprimento de onda de $660 \mathrm{~nm}$ (Miller, 1959).

A atividade proteolítica de CMCase foi determinada de acordo com Siqueira et al. (2010), utilizando-se $100 \mu \mathrm{L}$ de carboximetilcelulose $(1 \%)$ e $50 \mu \mathrm{L}$ da alíquota retirada do meio fermentativo. Os tubos com essa mistura foram incubados a $50^{\circ} \mathrm{C}$, por 30 minutos, e retirados para adição de $300 \mu \mathrm{L}$ de solução de ácido dinitrosalicílico (DNS), previamente preparada. Em seguida, a mistura foi fervida por dez minutos e 1,5 $\mathrm{mL}$ de água destilada foram adicionados. A leitura da absorbância foi feita em espectrofotômetro, com comprimento de onda de $540 \mathrm{~nm}$.

A atividade de protease foi determinada de acordo com Merheb et al. (2007), com modificações. Tubos com a mistura de $200 \mu \mathrm{L}$ do extrato enzimático, $400 \mu \mathrm{L}$ de caseína $(0,5 \%)$ e $400 \mu \mathrm{L}$ tampão acetato $\left(0,3 \mathrm{~mol} . \mathrm{L}^{-1}\right.$ de acetato de sódio: $1 \mathrm{~mol} . \mathrm{L}^{-1}$ de ácido acético), $\mathrm{pH} 5,5$, foram incubados, a $35^{\circ} \mathrm{C}$, por 30 minutos, e retirados para adição de $1 \mathrm{~mL}$ de ácido tricloroacético (10\%), a fim de parar a reação. A mistura reacional foi centrifugada a $4000 \mathrm{rpm}$, por cinco minutos e, em seguida, foi procedida a leitura de absorbância em espectrofotômetro, com comprimento de onda de $280 \mathrm{~nm}$.

Os ensaios de atividade de todas as enzimas foram conduzidos em triplicatas, havendo ainda o preparo de uma reação controle, que consistiu em mesmo tratamento peculiar a cada enzima, substituindo-se, porém, o extrato bruto enzimático por água destilada. A unidade das atividades enzimáticas (U.mg ${ }^{-1}$ ) foi definida como a quantidade de enzima capaz de liberar $1 \mu \mathrm{mol}$ de produto (açúcares redutores/aminoácidos) por segundo, por grama de amostra (polissacarídeos/proteínas), nas condições de reação, utilizando-se como curva padrão os monômeros de glicose e aminoácido.

As médias de atividades enzimáticas obtidas de cada tratamento, ao final de 24 horas, ao longo de quatro dias de cultivo, em estado sólido, em resíduos da colheita de soja, foram submetidas à análise de variância (ANOVA). A influência dos fatores, individualmente, e da interação entre eles, foi avaliada a partir do teste de regressão. $\mathrm{O}$ efeito do tempo sobre atividades das enzimas foi avaliado, independentemente dos tratamentos. Diferenças significativas $(\mathrm{p}<0,05)$ entre as médias foram determinadas pelo teste de Scott-Knott, utilizando-se o software Assistat (Silva \& Azevedo, 2009). 


\section{RESULTADOS E DISCUSSÃO}

O isolado fúngico Penicillium spp. LEMI A8221 apresentou habilidade metabólica para a produção de $\alpha$ e de $\beta$-amilase, de CMCase e de protease, independentemente dos tratamentos e tempos aplicados ao cultivo em estado sólido, utilizando-se resíduos da colheita de soja como substrato (Tabela 2).

Efeitos significativos dos tratamentos sobre as atividades das enzimas foram observados ao final de cada intervalo de tempo de cultivo em estado sólido (Tabela 2). Para a enzima $\alpha$-amilase, o T8 proporcionou maiores médias de atividade ( $\mathrm{p}<0,05)$, em todos os intervalos de tempo de cultivo $\left(0,02 ; 0,20 ; 0,19\right.$ e 0,14 U.mg-1 ${ }^{-1}$ em 24; 48; 72 e $96 \mathrm{~h}$, respectivamente). Maiores médias de atividade de â-amilase $(\mathrm{p}<0,05)$ foram registradas para T4 $(0,07$ U.mg$\left.{ }^{1}\right)$, após $24 \mathrm{~h}$ de cultivo e, para T1, nos demais intervalos de tempo (0,13 U.mg-1 , em 48 h e 0,11 U.mg ${ }^{-1}$, em 72 e 96 h). O T4 proporcionou maiores médias de atividade de CMCase ( $\mathrm{p}<0,05)$ em, pelo menos, três intervalos de tempo de cultivo $\left(0,39 ; 0,32\right.$ e $0,42 \mathrm{U}_{\mathrm{mg}} \mathrm{m}^{-1}$, em 24; 48 e $72 \mathrm{~h}$, respectivamente). Para protease, maiores médias de atividade ( $\mathrm{p}<0,05)$ foram verificadas para T6 $(145,3 ; 143,3$; 147,0 e 140,4 U.mg-1 ${ }^{-1}$ em 24; 48; 72 e 96 h, respectivamente) e T8 $\left(145,4 ; 144,5 ; 145,1\right.$ e 143,1 U.mg ${ }^{-1}$, em 24; 48; 72 e 96 $\mathrm{h}$, respectivamente).

Os valores máximos de atividade amilolítica registrados neste estudo (0,02 U.mg ${ }^{-1}$ para $\alpha$-amilase e $0,13{\mathrm{U} . \mathrm{mg}^{-1}}^{-1}$ para $\beta$-amilase) foram inferiores aos obtidos por Spier $e t$ al. (2006) trabalhando com fungos como o Aspergillus

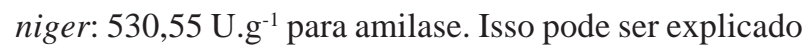
pelo elevado conteúdo de material lignocelulósico presente nos resíduos da colheita de soja, indicando a necessidade do fornecimento de material de mais fácil assimilação para promover a expressão ampliada de atividades amilolíticas, como farelo e grãos de soja. Kunamneni et al. (2005) obtiveram atividade enzimática de $534{\mathrm{U} . \mathrm{g}^{-1}}^{-1}$ de amilase a partir do cultivo do fungo Thermomyces lanuginosus, sob FES, em farelo de trigo, em temperaturas de incubação mais elevadas $\left(50^{\circ} \mathrm{C}\right)$, e constataram o incremento da produção de amilase com a adição de amido e de peptona ao meio fermentativo. Anto et al. (2006) observaram máxima produção de amilase $\left(271,2 \mathrm{U} \mathrm{g}^{-1}\right)$, utilizando farelo de trigo como substrato, com $\mathrm{pH}$ e temperatura ótimos de 5,0 e $55^{\circ} \mathrm{C}$, respectivamente, por FES, pelo fungo Aspergillus sp., e notaram aumento da produção da enzima pela adição de nitrogênio orgânico (extrato de levedura e peptona). Em relação à produção de CMCase, Jecu (2000) verificou atividade da endoglucanase igual a 14,8 U.mL ${ }^{-1}$ a partir do cultivo de A. niger, sob FES, em mistura de palha de trigo com farelo de trigo (9:1), na faixa de $\mathrm{pH} 4,5$ a 5,5 e temperatura ótima entre 28 a $34^{\circ} \mathrm{C}$. Por outro lado, Gawande \& Kamat (1999) obtiveram atividade máxima de CMCase igual a $0,53 \mathrm{U} \cdot \mathrm{mL}^{-1}$ por $A$. niger, sob FES, em casca de soja, a $35^{\circ} \mathrm{C}$, constando que este substrato foi resistente à sacarificação enzimática, por seu elevado conteúdo de lignina.

Os maiores valores de atividade enzimática registrados, neste estudo, foram para protease (131,7-147,0 U.mg-1). Esses resultados podem ser considerados satisfatórios, quando comparados com os de outros estudos. Rodrigues (2008) relatou um valor máximo de atividade de protease de 43,67 U.mg-1 ${ }^{-1}$ utilizando meios sintéticos para o cultivo de Penicillium aurantiogriseum, a $26^{\circ} \mathrm{C}$ e a $\mathrm{pH} 7 . \mathrm{Na}$ obtenção de extratos proteolíticos, por meio de FES, pelo fungo Aspergillus oryzae, utilizando farinha de peixe como substrato, García-Gómez et al. (2009) obtiveram uma atividade da protease alcalina (pH 10,0) de 120 U/L. Paris et al. (2010) verificaram que o maior valor de atividade para a protease (104,0 U.g $\mathrm{g}^{-1}$ ), a partir de FES, em farelo de soja, por Aspergillus casiellus, foi obtido com a maior adição de fonte inorgânica de carbono presente no meio fermentativo $(2 \%)$ e para a menor quantidade de fonte de nitrogênio $(0 \%)$ e menor valor de $\mathrm{pH}(3,0)$, evidenciando, neste caso, que a produção da enzima com característica ácida requer uma suplementação do meio, quando se utiliza farelo de soja como substrato.

A influência dos tratamentos sobre as atividades enzimáticas pode ser melhor compreendida a partir da avaliação da probabilidade dos efeitos $(\mathrm{P})$ das variáveis ambientais, individualmente, e da interação entre elas, para os dados referentes às médias das atividades das enzimas (Tabela 3). Verificou-se que as variáveis $\mathrm{pH}$ e temperatu$\mathrm{ra}$, individualmente, influenciaram significativamente $(\mathrm{p}<$ $0,05)$ apenas a atividade proteásica, uma vez que maior atividade média, ao final da fermentação, foi verificada para pH 6 e temperatura de $35^{\circ} \mathrm{C}$. A concentração de substrato igual a $90 \% \mathrm{p} / \mathrm{v}$ proporcionou os melhores resultados de atividade para CMCase e para protease, enquanto, para $\alpha$-amilase, maior média de atividade ocorreu com $70 \% \mathrm{p} / \mathrm{v}$ de substrato no meio de cultivo. A interação entre os fatores exerceu efeitos positivos significativos $(\mathrm{p}<0,05)$ para a atividade enzimática de pelo menos três das enzimas avaliadas, justificando os resultados anteriores quanto aos efeitos dos tratamentos sobre as atividades enzimáticas. Para $\alpha$-amilase, houve interação entre os fatores $\mathrm{pH}$ e concentração de substrato, ou seja, o aumento dos valores desses parâmetros dentro da faixa estudada incrementaria a produção de $\alpha$-amilase. Para $\beta$ amilase e protease, todas as interações entre os fatores foram significativas ( $\mathrm{p}<0,05)$ e apenas para CMCase, não foi observado o efeito significativo $(\mathrm{p}>0,05)$ da interação entre os fatores (Tabela 3). Sales et al. (2010) também observaram efeito significativo da interação entre $\mathrm{pH}$, concentração de substrato e temperatura de cultivo sobre a produção de exoglucanases por Aspergillus aculeatus, 
Tabela 2: Relação entre os tratamentos utilizados e a atividade amilásica, CMCásica e proteásica nos diferentes tempos de fermentação

\begin{tabular}{|c|c|c|c|c|c|c|c|c|}
\hline \multirow{3}{*}{ Tratamentos } & \multicolumn{4}{|c|}{$\alpha$-Amilase (U.mg ${ }^{-1}$ ) } & \multicolumn{4}{|c|}{$\boldsymbol{\beta}$-Amilase (U.mg-1) } \\
\hline & \multicolumn{4}{|c|}{ Tempo (horas) } & \multicolumn{4}{|c|}{ Tempo (horas) } \\
\hline & 24 & 48 & 72 & 96 & 24 & 48 & 72 & 96 \\
\hline T1 & $0,14 \pm 0,01 \mathrm{~d}$ & $0,14 \pm 0,02 \mathrm{~b}$ & $0,14 \pm 0,02 \mathrm{~b}$ & $0,20 \pm 0,01 \mathrm{a}$ & $0,05 \pm 0,01 \mathrm{~b}$ & $0,13 \pm 0,05 \mathrm{a}$ & $0,11 \pm 0,06 \mathrm{a}$ & $0,11 \pm 0,07 \mathrm{a}$ \\
\hline $\mathbf{T} 2$ & $0,15 \pm 0,02 \mathrm{~d}$ & $0,14 \pm 0,09 \mathrm{~b}$ & $0,14 \pm 0,02 \mathrm{~b}$ & $0,13 \pm 0,3 \mathrm{a}$ & $0,06 \pm 0,01 \mathrm{~b}$ & $0,06 \pm 0,01 \mathrm{~d}$ & $0,09 \pm 0,02 \mathrm{c}$ & $0,09 \pm 0,01 \mathrm{~b}$ \\
\hline T3 & $0,17 \pm 0,01 \mathrm{~b}$ & $0,19 \pm 0,04 \mathrm{a}$ & $0,19 \pm 0,05 \mathrm{a}$ & $0,13 \pm 0,02 \mathrm{~b}$ & $0,05 \pm 0,03 \mathrm{~b}$ & $0,06 \pm 0,02 \mathrm{~b}$ & $0,07 \pm 0,01 \mathrm{e}$ & $0,06 \pm 0,02 \mathrm{~d}$ \\
\hline T4 & $0,17 \pm 0,03 \mathrm{~b}$ & $0,19 \pm 0,01 \mathrm{a}$ & $0,13 \pm 0,01 \mathrm{~b}$ & $0,13 \pm 0,05 \mathrm{~b}$ & $0,07 \pm 0,01 \mathrm{a}$ & $0,08 \pm 0,01 \mathrm{~d}$ & $0,11 \pm 0,01 \mathrm{~b}$ & $0,08 \pm 0,03 \mathrm{~b}$ \\
\hline T5 & $0,16 \pm 0,01 \mathrm{c}$ & $0,12 \pm 0,01 \mathrm{c}$ & $0,13 \pm 0,01 \mathrm{~b}$ & $0,12 \pm 0,02 \mathrm{~b}$ & $0,05 \pm 0,01 \mathrm{~b}$ & $0,06 \pm 0,02 \mathrm{~d}$ & $0,06 \pm 0,01 \mathrm{e}$ & $0,07 \pm 0,03 \mathrm{c}$ \\
\hline T6 & $0,16 \pm 0,03 \mathrm{c}$ & $0,19 \pm 0,03$ a & $0,13 \pm 0,03 \mathrm{~b}$ & $0,13 \pm 0,02 \mathrm{~b}$ & $0,06 \pm 0,01 \mathrm{~b}$ & $0,08 \pm 0,03 \mathrm{~b}$ & $0,08 \pm 0,01 \mathrm{~d}$ & $0,08 \pm 0,02 \mathrm{~b}$ \\
\hline T7 & $0,17 \pm 0,03 \mathrm{~b}$ & $0,19 \pm 0,04 \mathrm{a}$ & $0,19 \pm 0,03 \mathrm{a}$ & $0,13 \pm 0,01 \mathrm{~b}$ & $0,05 \pm 0,03 \mathrm{~b}$ & $0,06 \pm 0,03 \mathrm{~d}$ & $0,06 \pm 0,01 \mathrm{e}$ & $0,06 \pm 0,03 \mathrm{~d}$ \\
\hline \multirow[t]{2}{*}{ T8 } & $0,02 \pm 0,02 \mathrm{a}$ & $0,20 \pm 0,01 \mathrm{a}$ & $0,19 \pm 0,01 \mathrm{a}$ & $0,14 \pm 0,2 \mathrm{a}$ & $0,05 \pm 0,01 \mathrm{~b}$ & $0,07 \pm 0,02 \mathrm{c}$ & $0,09 \pm 0,02 \mathrm{c}$ & $0,10 \pm 0,02 \mathrm{~b}$ \\
\hline & \multicolumn{4}{|c|}{ CMCase (U.mg ${ }^{-1}$ ) } & \multicolumn{4}{|c|}{ Protease (U.mg-1) } \\
\hline \multirow[t]{2}{*}{ Tratamentos } & \multicolumn{4}{|c|}{ Tempo (horas) } & \multicolumn{4}{|c|}{ Tempo (horas) } \\
\hline & 24 & 48 & 72 & 96 & 24 & 48 & 72 & 96 \\
\hline T1 & $0,29 \pm 0,06 \mathrm{c}$ & $0,35 \pm 0,05 \mathrm{~d}$ & $0,40 \pm 0,08 \mathrm{e}$ & $0,38 \pm 0,05 \mathrm{e}$ & $134,2 \pm 0,3 \mathrm{~d}$ & $141,0 \pm 0,5 \mathrm{~d}$ & $136,5 \pm 0,5 \mathrm{f}$ & $135,8 \pm 0,1 \mathrm{e}$ \\
\hline $\mathbf{T} 2$ & $0,34 \pm 0,04 \mathrm{~b}$ & $0,38 \pm 0,03 \mathrm{c}$ & $0,44 \pm 0,07 \mathrm{c}$ & $0,46 \pm 0,09 \mathrm{c}$ & $134,6 \pm 1,2 \mathrm{~d}$ & $132,0 \pm 0,1 \mathrm{f}$ & $132,3 \pm 0,2 \mathrm{~g}$ & $131,7 \pm 0,2 \mathrm{f}$ \\
\hline T3 & $0,37 \pm 0,08$ a & $0,34 \pm 0,4 \mathrm{~d}$ & $0,39 \pm 0,06 \mathrm{e}$ & $0,36 \pm 0,05 \mathrm{e}$ & $136,8 \pm 1,2 \mathrm{c}$ & $141,0 \pm 0,2 \mathrm{~b}$ & $136,5 \pm 0,2 \mathrm{f}$ & $135,8 \pm 0,1 \mathrm{e}$ \\
\hline T4 & $0,39 \pm 0,05$ a & $0,42 \pm 0,1 \mathrm{a}$ & $0,52 \pm 0,08 \mathrm{a}$ & $0,43 \pm 0,2 \mathrm{~d}$ & $143,7 \pm 0,2 \mathrm{a}$ & $138,0 \pm 0,1 \mathrm{~d}$ & $142,2 \pm 0,6 \mathrm{c}$ & $142,4 \pm 0,2 \mathrm{a}$ \\
\hline T5 & $0,30 \pm 0,1 \mathrm{c}$ & $0,32 \pm 0,09 \mathrm{e}$ & $0,36 \pm 0,1 \mathrm{f}$ & $0,64 \pm 0,1 \mathrm{a}$ & $140,4 \pm 0,2 \mathrm{~b}$ & $139,2 \pm 0,9 \mathrm{c}$ & $137,5 \pm 0,1 \mathrm{e}$ & $137,5 \pm 0,5 \mathrm{~d}$ \\
\hline T6 & $0,29 \pm 0,1 \mathrm{c}$ & $0,43 \pm 0,1 \mathrm{a}$ & $0,42 \pm 0,01 \mathrm{~d}$ & $0,43 \pm 0,1 \mathrm{~d}$ & $145,3 \pm 0,3 \mathrm{a}$ & $143,3 \pm 0,3 \mathrm{a}$ & $147,0 \pm 0,3$ a & $140,4 \pm 0,5 \mathrm{~b}$ \\
\hline T7 & $0,30 \pm 0,09 \mathrm{c}$ & $0,34 \pm 0,1 \mathrm{~d}$ & $0,36 \pm 0,1 \mathrm{f}$ & $0,35 \pm 0,03 \mathrm{f}$ & $142,2 \pm 0,2 \mathrm{~b}$ & $136,6 \pm 0,2 \mathrm{e}$ & $139,4 \pm 0,1 \mathrm{~d}$ & $138,8 \pm 0,4 \mathrm{c}$ \\
\hline T8 & $0,26 \pm 0,1 \mathrm{~d}$ & $0,40 \pm 0,02 \mathrm{~b}$ & $0,48 \pm 0,04 \mathrm{~b}$ & $0,49 \pm 0,02 \mathrm{~b}$ & $145,4 \pm 0,2 \mathrm{a}$ & $144,5 \pm 1,0 \mathrm{a}$ & $145,1 \pm 0,1 \mathrm{~b}$ & $143,1 \pm 0,2 \mathrm{a}$ \\
\hline
\end{tabular}

Os valores referem-se à média de três determinações de atividade das enzimas $\beta$ e $\beta$-amilase, CMCase e protease, para cada intervalo de tempo de cultivo em estado sólido em resíduos da colheita de soja. Médias seguidas por letras iguais, na coluna, não diferem entre si $(\mathrm{P}>0,05)$ pelo teste de Scott Knott.

Tabela 3: Probabilidade dos efeitos $(\mathrm{P})$ para os dados referentes às médias das atividades das enzimas amilase dextrinizante, $\beta$-amilase, $\mathrm{CMCase}$ e protease, obtidas ao longo do processo fermentativo, em estado sólido, com resíduos da colheita da soja como substrato

\begin{tabular}{|c|c|c|c|c|c|c|c|c|c|c|}
\hline \multirow{2}{*}{ Atividade Enzimática } & \multicolumn{2}{|c|}{ pH } & \multicolumn{2}{|c|}{ Temperatura $\left({ }^{\circ} \mathrm{C}\right)$} & \multicolumn{2}{|c|}{ Substrato $(\%)$} & \multirow{2}{*}{ I1 } & \multirow{2}{*}{$\mathbf{I} 2$} & \multirow{2}{*}{ I3 } & \multirow{2}{*}{ I4 } \\
\hline & 5 & 6 & 30 & 35 & 70 & 90 & & & & \\
\hline Amilase Dextrinizante & $0,14 \mathrm{a}$ & $0,14 \mathrm{a}$ & $0,13 \mathrm{a}$ & $0,14 \mathrm{a}$ & $0,15 \mathrm{a}$ & $0,14 \mathrm{~b}$ & NS & $\mathrm{P}<0,05$ & NS & NS \\
\hline Amilase Sacarificante & $0,07 \mathrm{a}$ & $0,06 \mathrm{a}$ & $0,07 \mathrm{a}$ & $0,06 \mathrm{a}$ & $0,06 \mathrm{a}$ & $0,07 \mathrm{a}$ & $\mathrm{P}<0,05$ & $\mathrm{P}<0,05$ & $\mathrm{P}<0,05$ & $\mathrm{P}<0,05$ \\
\hline CMCase & $0,39 \mathrm{a}$ & $0,38 \mathrm{a}$ & $0,38 \mathrm{a}$ & $0,38 \mathrm{a}$ & $0,36 \mathrm{~b}$ & $0,41 \mathrm{a}$ & NS & NS & NS & NS \\
\hline Protease & $136 \mathrm{~b}$ & $141 \mathrm{a}$ & $137 \mathrm{~b}$ & $140 \mathrm{a}$ & $137 \mathrm{~b}$ & $140 \mathrm{a}$ & $\mathrm{P}<0,05$ & $\mathrm{P}<0,05$ & $\mathrm{P}<0,05$ & $\mathrm{P}<0,05$ \\
\hline
\end{tabular}

N NS: não significativo; Efeito das interações: $\mathrm{pH}$ x temperatura (I1); $\mathrm{pH}$ x concentração de substrato (I2); temperatura x concentração de substrato (I3); $\mathrm{pH}$ x temperatura $\mathrm{x}$ concentração de substrato (I4).

$\sigma$ Médias seguidas de mesmas letras não diferem entre si $(\mathrm{P}>0,05)$ pelo teste de Scott Knott. 
sob cultivo em estado sólido, em bagaço de cana-de-açúcar. Os autores constataram que quanto maiores a concentração de substrato e a temperatura de cultivo, maior foi a atividade enzimática, ou seja, o efeito da interação desses fatores foi positivo. $\mathrm{O} \mathrm{pH}$, no entanto, teve efeito negativo: a mais alta atividade de exoglucanase foi de 0,17 U.mL $\mathrm{m}^{-1}$ em pH 6,0, concentração de substrato $1,5 \%$ e temperatura de cultivo a $40^{\circ} \mathrm{C}$.

$\mathrm{O}$ fato de a variável $\mathrm{pH}$ ter influenciado somente a atividade proteásica pode estar relacionado com a sensibilidade desta enzima a uma possível variação dos valores de $\mathrm{pH}$, ao longo do processo fermentativo, uma vez que o ajuste do $\mathrm{pH}$ foi realizado apenas no início do processo. Da mesma forma, a atividade proteásica, neste estudo, foi sensível aos valores de temperatura testados, monstrando que essa enzima foi susceptível ao acúmulo de calor metabólico gerado, que ocorre, normalmente em cultivo em estado sólido, decorrente da dificuldade de mistura do meio sólido, além da baixa condutividade térmica que a maioria dos substratos apresenta (Hölker \& Lenz, 2005).

Das variáveis ambientais, individualmente avaliadas, a concentração do substrato se mostrou a mais crítica. Esses resultados podem ser justificados não pelo papel nutricional dos substratos, mas, principalmente, pela umidade que cada tratamento proporcionou ao desenvolvimento do fungo e, consequentemente, à produção de

A

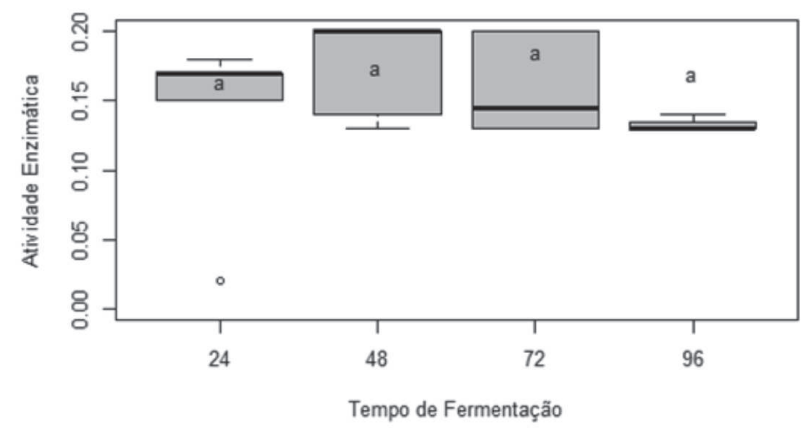

c

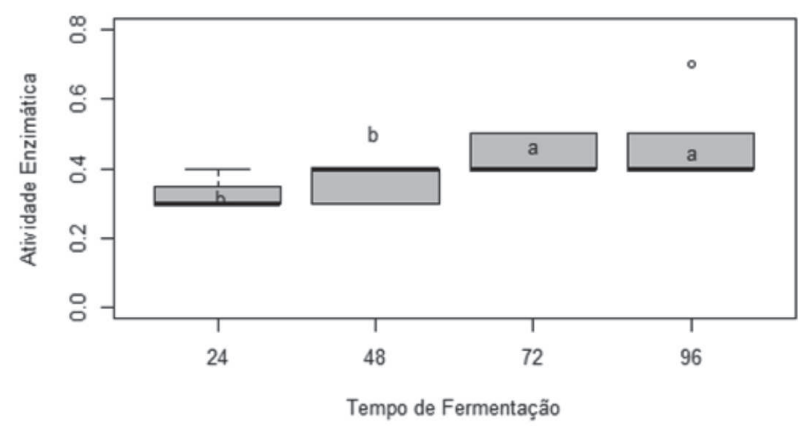

enzimas. Nos tratamentos com $90 \%$ p/v de substrato, a percentagem de água na massa total do meio era menor que nos tratamentos com $70 \%$ p/v de substrato. Segundo Gervais \& Molin (2003), a umidade é o fator ambiental que mais influencia o processo de cultivo em estado sólido, sendo que um nível de umidade muito alto resulta em diminuição da porosidade, baixa difusão de oxigênio, aumento do risco de contaminação, redução do volume de gás e redução de troca gasosa. Por outro lado, reduzidos níveis de umidade levam a um menor grau de crescimento, em relação ao ótimo, e a baixa percentagem de substrato realmente utilizado (Lonsane et al., 1985).

O tempo de fermentação foi avaliado como uma variável independente dos tratamentos, conforme ilustrado na Figura 1. Para $\alpha$-amilase e para protease, os intervalos de tempo não influenciaram significativamente $(\mathrm{p}>0,05)$ a atividade das enzimas (Figura 1A e D). O tempo de cultivo reduzido, alcançado neste trabalho, pode representar uma melhoria especial para técnicas de fermentação que utilizam Penicillium spp., uma vez que o custo da produção de enzimas é proporcional ao tempo de incubação (Garcia et al., 2015). Para $\beta$-amilase e para CMCase, foi verificado efeito significativo $(\mathrm{p}<0,05)$ do tempo, sendo as primeiras horas de fermentação as que proporcionaram menor atividade dessas enzimas (Figura 1B e C). A elevação da atividade após $48 \mathrm{~h}$ pode ser explicada pela baixa disponibilidade de açúcares redutores da matéria-prima, neces-

B

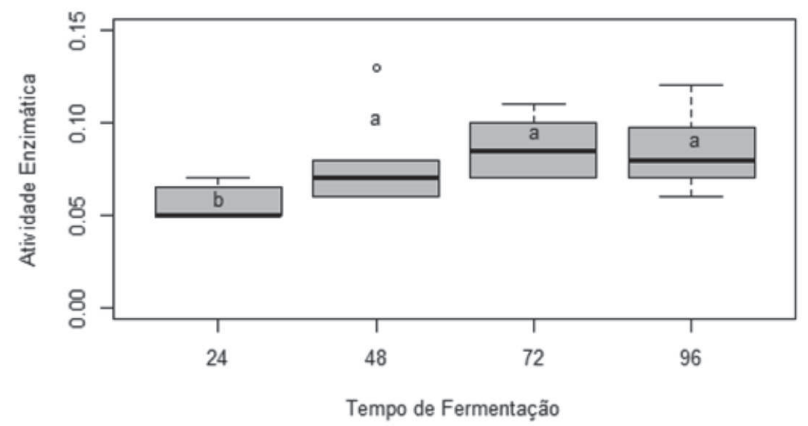

D

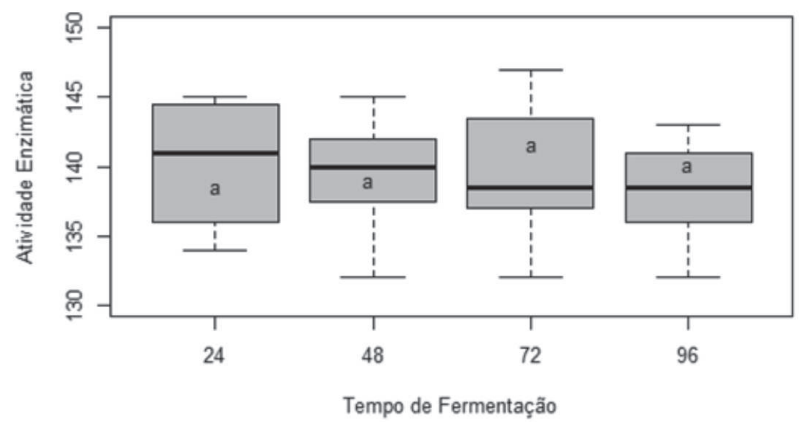

Figura 1: Efeito dos intervalos de tempo (horas) do processo de fermentação, em estado sólido, sobre as atividades enzimáticas $\left(\mathrm{U} . \mathrm{mg}^{-1}\right)$, independentemente dos tratamentos de $\mathrm{pH}$, temperatura e concentração de substrato. A: $\alpha$-amilase; B: $\beta$-amilase; C: CMCase e D: protease. Médias seguidas de mesmas letras não diferem entre si (P > 0,05) pelo teste de Scott Knott. 
sários para o desenvolvimento do micro-organismo, levando ao estímulo do mecanismo de expressão das enzimas necessárias para geração de açúcares simples (Whitaker, 1994).

\section{CONCLUSÕES}

Os resíduos da colheita de soja se mostraram-se como o substrato alternativo interessante para ser empregado em processo fermentativo. No entanto, é válido considerar a separação dos componentes do resíduo, a fim de serem aplicados, individualmente, como substratos em cultivo, em estado sólido, para produção de enzimas, em pesquisas futuras. O isolado Penicillium spp. LEMI A8221 pode ser considerado promissor agente biológico com aplicação industrial, especialmente para produção de protease, uma vez que esse fungo apresentou satisfatória eficiência na conversão do substrato em produto. Além disso, o isolado fúngico exigiu condições de processo pouco complexas e foi hábil, metabolicamente, para utilizar resíduos da colheita de soja como única fonte de carbono. Apesar de este estudo ter sido realizado em escala de bancada, os resultados obtidos são entendidos como ponto de partida para o escalonamento do processo.

\section{AGRADECIMENTOS}

Os autores agradecem ao Conselho Nacional de Desenvolvimento Científico e Tecnológico (CNPq), à Fundação de Amparo à Pesquisa do Estado da Bahia (Fapesb) e à Universidade Federal da Bahia (UFBA), pelo financiamento de estudos e projetos.

\section{REFERÊNCIAS}

Anto H, Trivedi UP \& Patel KC (2006) Glucoamylase production by solid-state fermentation using rice flake manufacturing waste products as substrate. Bioresource Technology, 97:1161-1166.

Brijwani K \& Vadlani PV (2011) Cellulolytic enzymes production via solid-state fermentation: Effect of pretreatment methods on physicochemical characteristics of substrate. Enzyme Research, 2011:01-10.

Carvalho FC de (1992) Disponibilidade de resíduos agro-industriais e do beneficiamento de produtos agrícolas. In: Simpósio de utilização de subprodutos agroindustriais e resíduos de colheita na alimentação de ruminantes, São Carlos. Anais, Embrapa. 349:07-27.

Couto SR \& Sanromán MA (2005) Application of solid-state fermentation to ligninolytic enzyme production. Biochemical Engineering Journal, 22:211-219.

García-Gómez MJ, Huerta-Ochoa S, Loera-Corral O \& PradoBarragán LA (2009) Advantages of a proteolytic extract by Aspergillus oryzae from fish flour over a commercial proteolytic preparation. Food Chemistry, 112:604-608.

Garcia NLF, Santos FRS, Gonçalves FA, Paz MF da, Fonseca GG \& Leite RSR (2015) Production of â-glucosidase on solid-state fermentation by Lichtheimia ramosa in agroindustrial residues: Characterization and catalytic properties of the enzymatic extract. Electronic Journal of Biotechnology 18:314-319.
Gawande PV \& Kamat MY (1999) Production of Aspergillus xylanase by lignocellulosic waste fermentation and its application. Journal of Applied Microbiology, 87:511-519.

Gervais P \& Molin P (2003) The role of water in solid-state fermentation. Biochemical Engineering Journal, 13:85-101.

Hölker U \& Lenz J (2005) Solid-state fermentation - are there any biotechnological advantages? Current Opinion in Microbiology, 8:301-306.

Jecu L (2000) Solid state fermentation of agricultural wastes for endoglucanase production. Industrial Crops and Products, 11:0105 .

Kim DM, Cho EJ, Kim JW, Lee YW \& Chung HJ (2014) Production of cellulases by Penicillium sp. in a solid- state fermentation of oil palm empty fruit bunch. African Journal of Biotechnology, $13: 145-155$.

Kunamneni A, Permaul K \& Singh S (2005) Amylase Production in Solid State Fermentation by the Thermophilic Fungus Thermomyces lanuginosus. Journal of Bioscience and Bioengineering, 100:168-171.

Lonsane BK, Ghildyal NP, Budiatman S \& Ramakrishna SV (1985) Engineering aspects of solid state fermentation. Enzyme and Microbial Technology, 7:258-265.

Merheb CW, Cabral H, Gomes E \& Da-Silva R (2007) Partial characterization of protease from thermophilic fungus Thermoascus aurantiacus and its hydrolytic activity on bovine casein. Food Chemistry, 104:127-131.

Miller GL (1959) Use of dinitosalicylic acid reagent for the determination of reducing sugar. Analytical Chemistry, 31:426428.

Paris L de, Scheufele FB, Júnior AT, Guerreiro TL \& Hasan SDM (2010) Estudo do crescimento de A. casiellus em farelo de soja convencional para produção de enzimas. Estudos tecnológicos, $6: 1: 22-35$.

Paris L de, Scheufele FB, Júnior AT, Guerreiro TL \& Hasan SDM (2012) Produção de complexos enzimáticos por A. niger a partir de soja por fermentação em estado sólido. Acta Scientiarum Technology, 34:193-200.

Rodrigues PMB (2008) Produção de protease pelo Penicillium aurantiogriseum URM4622. Dissertação de Mestrado. Universidade Federal de Pernambuco, Pernambuco. 58p.

Saidelles APF, Senna AJT, Kirchner R \& Bitencourt G (2012) Gestão de resíduos sólidos na indústria de beneficiamento de arroz. Revista Eletrônica em Gestão, Educação e Tecnologia Ambiental, 5:904-916.

Sales MR, de Moura RB, Porto TS, de Macedo GR \& Porto ALF (2010) Variáveis que influenciam a produção de celulases e xilanase por espécies de Aspergillus. Pesquisa Agropecuária Brasileira, 45:1290-1296.

Sánchez C (2009) Lignocellulosic residues: Biodegradation and bioconversion by fungi. Biotechnology Advances 27:185-194.

Saxena R \& Singh R (2011) Amylase production by solid-state fermentation of agro-industrial wastes using Bacillus sp. Brazilian Journal of Microbiology, 42:1334-1342.

Sena AR, Koblitz MGB, Goes Neto A \& Uetanabaro APT (2006) Seleção de fungos do semi-árido baiano secretores de hidrolases de interesse em alimentos. Sitientibus, 35:91-98.

Silva FASE \& Azevedo CAV (2009) Principal components analysis in the software assistat-statistical assistance. In: $7^{\text {th }}$ World Congress on Computers in Agriculture, Reno. Proceedings, ASABE. p.01-05. 
Silva EM, Machuca A \& Milagres AMF (2005) Effect of cereal brans on Lentinula edodes growth and enzyme activities during cultivation on forestry waste. Letters in Applied Microbiology, 40:283-288.

Siqueira FG, Siqueira EG, Jaramillo PMD, Silveira MHL, Andreaus J, Couto FA, Batista LR \& Filho EXF (2010) The potential of agro-industrial residues for production of holocellulases from filamentous fungi. International Biodeterioration and Biodegradation, 64:20-26.

Sohail M, Naseeb S, Sherwani SK, Sultana S, Aftab S, Shahzad S, Ahmad A \& Khan SA (2009) Distribution of hydrolytic enzymes among native fungi: Aspergillus the pre-dominant genus of hhydrolase producer. Pakistan Journal of Botany, 41:2567-2582.

Spier MR, Woiciechowski AL, Vandenberghe L \& Soccol CR (2006) Production and Characterization of amylases by Aspergillus niger under solid state fermentation using agro industrials products. International Journal of Food Engineering, 2:01-19.
Sukumaran RK, Singhania RR \& Pandey A (2005) Microbial celulases - Production, applications and challenges. Journal of Scientiic \& Industrial Research, 64:832-844.

Thakur SA, Nemade SN \& Sharanappa A (2015) Solid state fermentation of overheated soybean meal (Waste) for production of protease using Aspergillus Oryzae. International Journal of Innovative Research in Science, Engineering and Technology, 4:18456-18461.

Whitaker JR (1994) Principles of enzymology for the food sciences. $2^{\mathrm{a}}$ ed. New York, Marcel Dekker. 648p.

Wisniewski AC, de Almeida MAL, Palma MP \& Tavares LBB (2010) Produção de enzimas amilolíticas por Macrocybe titans em resíduo do processamento de cerveja. Revista Brasileira de Biociências, 8:285-293. 